\title{
Mathematical Models for Estimating Effective Diffusion Parameters of Spherical Drug Delivery Devices
}

\author{
Song Wang*, Shalela Mohd Mahali*, Andrea McGuiness** and Xia Lou**† \\ *School of Mathematics \& Statistics, The University of Western Australia \\ 35 Stirling Highway, Crawley WA 6009, Australia \\ **Nanochemistry Research Institute \& Department of Chemical Engineering, Curtin \\ University of Technology \\ Kent Street, Bentley WA 6102, Australia
}

${ }^{\dagger}$ Corresponding Author. Tel.: +61 8 92661682; Fax: +61 8 92662168; Email: 


\section{$\underline{\text { x.lou@ curtin.edu.au }}$}

Abstract: Mathematical modeling of drug delivery is of increasing academic and industrial importance in many aspects. In this paper we propose an optimization approach for the estimation of the parameters characterizing the diffusion process of a drug from a spherical porous polymer device to an external finite volume. The approach is based on a nonlinear least-squares method and a novel mathematical model which takes into consideration both boundary layer effect and initial burst phenomenon. An analytical solution to the model is derived and a formula for the ratio of the mass released in a given time interval and the total mass released in infinite time is also obtained. The approach has been tested using experimental data of the diffusion of prednisolone 21-hemisuccinate sodium salt from spherical devices made of porous poly(2-hydroxyethyl methacrylate) hydrogels. The effectiveness and accuracy of the method are well demonstrated by the numerical results. The model was used to determine the diffusion parameters including the effective diffusion coefficient of the drug from a series of devices that vary in both the porous structure and the drug loading levels. The computed diffusion parameters are discussed in relation to the physical properties of the devices.

Keywords: Controlled drug delivery, effective diffusion coefficient, optimization, diffusion 
equation, hydrogels 


\section{Introduction}

Mathematical modeling of drug delivery is a field of significant academic and economic importance. This is true not only in the biopharmaceutical disciplines [1], but also in the increasingly active tissue engineering research field where the development of three dimensional scaffolds meeting the requirements of cell migration, tissue growth, and the transportation of nutritious chemicals such as growth factors is still a challenge [2]. An ideal delivery requires a device to supply and release therapeutic agents to a desired location with a precise therapeutic dose for a prolonged period of time [3]. The controllability of the delivery is dependent on many variables. These include the transport properties and the dosage of the drugs; the physiochemical and structural properties, the dimensions and geometry as well as the release mechanisms of the drug delivery systems. Effectively predicting these parameters and ultimately optimizing the design of a drug delivery system using mathematical approaches can significantly reduce manufacturing costs of both new and existing products $[1]$.

On the other hand, mathematical tools, particularly numerical partial differential equation and optimization techniques have been used successfully and extensively in optimum designs of many engineering devices such as semiconductor devices (cf., for example, [4-7]). Despite the success of these techniques in many areas, reports on the systematic use of advanced mathematical tools in the design of controlled drug delivery devices are limited in the open literature, except for some simple models with known analytical solutions of the diffusion equation (cf., for example, [8-10]). Our previous studies have shown that the mathematical approach is indeed useful in interpreting experimental data and establishing the relationship between the drug release characteristics and the material structures [11, 12]. This paper extends our studies on the parameter estimation of controlled drug delivery systems of a disk 
geometry to a spherical geometry using similar mathematical tools. The drug delivery systems used in our studies are based on a porous matrix made of poly(2-hydroxyethyl methacrylate) (PHEMA) hydrogels.

PHEMA is well known for its biomedical applications as contact lenses, intraocular lenses and cardiovascular implants $[13,14]$. Materials based on PHEMA absorb large amounts of water without dissolving, and in their swollen state they behave like typical gels. Therefore, the term hydrogels is commonly employed for them. In most applications PHEMA hydrogels refer to the crosslinked polymers produced by bulk polymerization which are transparent and contain a homogeneous polymer matrix containing pores measured in nanometers. Although polymers of this type allow the diffusion of various solutes, their transport properties are limited by effective mean pores, or mesh diameters, within the polymer. They are more suited for such applications as contact lenses, in which a combination of optical clarity and limited diffusive characteristics is required [15].

Various methods can be used to increase the effective pore sizes of PHEMA. One of the most convenient methods is to polymerise the HEMA monomer in the presence of water above a critical level (reportedly 40-45\%) (cf. [11] and the references quoted). The materials produced in the presence of water possess high water content and pores ranging from several to hundreds of microns. The biomedical applications of porous PHEMA materials include a novel design of an artificial cornea and an orbit implant in which the porous PHEMA skirt allows host cells and tissue to grow into the device therefore preventing extrusion of the implants [16-18]. Our recent studies show that the porous PHEMA hydrogels represent a significant advance over the non-porous types in the drug delivery applications with a much higher drug loading capacity. The loading of drugs can be achieved in ambient conditions 
with very simple means, less concerning about the drug stability $[11,12]$. To achieve an optimal design of such a device, we have been investigating mathematical models for (i) extracting the effective diffusion coefficient of a selected drug; and (ii) further establishing the relationship between the diffusion characteristics and various parameters of the drug delivery system including the drug loading level, the porosity and the geometry of the polymer matrix.

It is worth mentioning that drug delivery from porous PHEMA is diffusion driven. In a diffusion-controlled device, the delivery of drugs is largely dependent on the diffusion property of the drug in a constructed device, which is often characterized by the effective diffusion coefficient of the drug in the material. The effective diffusion coefficient of a drug delivery system is a measure of the diffusion process of a drug through a selected system over a period of time. It is determined mainly by the properties of a polymer matrix and the interactions, if any, between the drug and the polymer matrix. For a given device, drug release profiles from the device into a finite volume during a period of time can be determined through laboratory experiments. The estimation of the effective diffusion coefficient of the drug during the process involves two tasks. One task is to set up a mathematical model for the diffusion process of the device and the other is to numerically estimate the effective diffusion coefficient based on the model and some given information such as experimentally observed drug release data.

In general, a diffusion process is governed by a diffusion equation with appropriate initial and boundary conditions. However, solving such a diffusion problem analytically is very difficult. Analytical and approximate solutions to several simple models can be found in [1, 19-22]. Some widely used models such as those in [22] are based on the assumption that the liquid in 
the diffusion region is 'well-stirred', i.e., the concentration of the substance in the liquid is uniform which is not always true. In practice, even if the liquid is 'well stirred' the magnitude of the flow velocity on the boundary of the device should be zero due to the so-called 'noslip' boundary condition. Therefore, there exists a region, called a boundary layer, near the boundary of the device in which the magnitude of velocity varies from zero to some positive value. As a result, the substance concentration is non-uniform in the boundary layer. Furthermore, excessive drugs may be left on the surface of the device which causes a higher concentration on the surface than in the subsurface of the device. It is also possible that the drug concentration on the surface is lower than that in the subsurface of the device if the device is pre-washed prior to a drug release experiment. Both of these cases may lead to an initial phase of the drug release that is different from the rest of the process. Therefore, it is desirable to determine an effective critical time separating the two phases and to extract the effective diffusion coefficients for the two phases.

Once a diffusion model has been established, one needs to determine the effective diffusion coefficient using the model. A classical 'trial-and-error' process is neither optimal nor automatic. In our previous work [23], we have proposed a model for the estimation of effective diffusion coefficients and other critical parameters of PHEMA devices of a 2D disc geometry. The model was used in conjunction with a nonlinear least-squares method. Unlike existing ones such as those in [22], this model can handle both the initial burst and boundary layer effects. In the present work, we extend the techniques in [23] to devices of a spherical geometry (Figure 1). We first propose a basic mathematical model governing the diffusion process of a drug from a spherical device into a finite volume. The model is then further developed to include both the initial burst and the boundary layer effects. Analytical solutions to these mathematical problems are then obtained to provide explicit expressions for the total 
mass diffused from the device into the external volume in a given period of time. The unknown parameters including the effective diffusion coefficients, the width of the boundary layer and the critical time in the models are determined by an optimization technique. Six porous PHEMA spherical devices that contain various pore structures and different levels of prednisolone 21-hemisuccinate sodium salt, a commonly used anti inflammation drug, are then prepared. The drug release experiments are conducted and the acquired data are used to test the mathematical models. The full model is finally used to determine the diffusion parameters including the critical time for the initial burst of drugs, the effective boundary layer, and the effective diffusion coefficient of the drug from these devices. The drug diffusion characteristics are discussed in relation to the physical properties of the devices.

Figure 1. Schematic illustration of a porous PHEMA spherical device.

\section{The mathematical methods}

\subsection{The basic model and its analytical solution}

We first consider a spherical device with radius $r_{1}$ pre-loaded with an amount of drug, $M^{0}$. Assuming, 1) the device is placed in a sphere container of radius $r_{2}$ filled with water so that 
the device and the container are concentric, as depicted in Figure 2; and 2) the release process is diffusion-dominant and radial because of symmetry, i.e. the concentration of drug in liquid is uniform for a fixed $r$, the diffusion process of this problem is governed by the following diffusion equation in spherical coordinates:

$$
\begin{aligned}
& \frac{\partial C(r, t)}{\partial t}=D\left(\frac{\partial^{2} C(r, t)}{\partial r^{2}}+\frac{2}{r} \frac{\partial C(r, t)}{\partial r}\right), 0<r<r_{2}, t>0, \\
& \frac{\partial C\left(r_{2}, t\right)}{\partial r}=0, t>0, \\
& C(r, 0)=H(r),
\end{aligned}
$$

where $D$ is a constant and $C(r, t)$ is the unknown concentration.

Figure 2: A sphere device with radius $r_{1}$ placed in a container with radius $r_{2}$.

We assume that at $t=0$, the concentration is uniform in the device and zero in liquid, i.e.,

$$
H(r)=\left\{\begin{array}{cc}
M^{0} / V_{d}, & 0<r<r_{1}, \\
0, & r_{1}<r<r_{2},
\end{array}\right.
$$

where $V_{d}=4 \pi r_{1}^{3} / 3$ is the volume of the device. To solve this problem, we use the technique of separation of variables as outlined below. 
Let $C(r, t)=u(t) v(r)$. Eq. (2.1) then becomes

$$
u^{\prime} v=D\left(u v^{\prime \prime}+\frac{2}{r} u v^{\prime}\right)=D u\left(v^{\prime \prime}+\frac{2}{r} v^{\prime}\right)
$$

From this we have

$$
\frac{u^{\prime}}{D u}=\frac{v^{\prime \prime}+\frac{2}{r} v^{\prime}}{v}=-\lambda,
$$

where $\lambda>0$ is a constant to be determined. The above expression contains two equations:

$$
\begin{aligned}
& u^{\prime}+\lambda D u=0, \\
& v^{\prime \prime}+\frac{2}{r} v^{\prime}+\lambda v=0 .
\end{aligned}
$$

Eq. (2.5) has the (fundamental) solution $u=e^{-\lambda D t}$ and Eq. (2.6) is a Bessel's equation of the form

$$
y^{\prime \prime}+(d-1) y^{\prime} / x+\left(\lambda-\mu / x^{2}\right) y=0
$$

with $d=3$ and $\mu=0$. The fundamental solution to this equation is (cf., for example, [25], p.231)

$$
v(r)=j_{0}(r \sqrt{\lambda})
$$

where $j_{0}(z)=\frac{\sin z}{z}$ is the 0 th order spherical Bessel function. Therefore, the solution of $(2.1)$ is of the form

$$
C_{\lambda}(r, t)=j_{0}(r \sqrt{\lambda}) e^{-\lambda D t}
$$

where $\lambda$ is a parameter called the eigenvalue of the problem. To determine $\lambda$, we apply the boundary condition (2.2) to (2.7) to get

$$
\frac{\partial C_{\lambda}\left(r_{2}, t\right)}{\partial r}=j_{0}^{\prime}\left(r_{2} \sqrt{\lambda}\right) \sqrt{\lambda} e^{-\lambda D t}=0 .
$$

This implies $j_{0}^{\prime}\left(r_{2} \sqrt{\lambda}\right)=0$. Let $\alpha_{n}>0$ be such that 


$$
j_{0}^{\prime}\left(\alpha_{n}\right)=\frac{\alpha_{n} \cos \alpha_{n}-\sin \alpha_{n}}{\alpha_{n}^{2}}=0 \quad \text { for } n=1,2, \ldots
$$

Then, we have $r_{2} \sqrt{\lambda}=\alpha_{n}$ or

$$
\lambda_{n}=\alpha_{n}^{2} / r_{2}^{2} \quad \text { for } n=1,2, \ldots
$$

Substituting the above $\lambda_{n}$ into (2.7) gives

$$
C_{\lambda_{n}}(r, t)=j_{0}\left(\frac{r \alpha_{n}}{r_{2}}\right) e^{-D \alpha_{n}^{2} t / r_{2}^{2}} .
$$

This is a solution to (2.1) for each $n=1,2, \ldots$.

When $\lambda=0,(2.6)$ has the general solution

$$
v=\frac{B_{0}}{r}+A_{0}
$$

with additive constants $A_{0}$ and $B_{0}$. (Note $\alpha_{0}=0$ is also a root of (2.8) and thus $\lambda=0$ is the eigenvalue corresponding to this root.) Thus, (2.9) represents the steady-state solution to (2.1)-(2.3). Applying the boundary condition (2.2) to (2.9) gives $B_{0}=0$. Therefore, combining the fundamental solutions to (2.5) and (2.6) and using the superposition principle, we have the following series solution to (2.1):

$$
C(r, t)=\sum_{n=0}^{\infty} A_{n} j_{0}\left(\frac{\alpha_{n} r}{r_{2}}\right) e^{-D \alpha_{n}^{2} t / r_{2}^{2}},
$$

where $A_{n}$ 's are coefficients to be determined. (Recall that $\alpha_{0}=0$ and $j_{0}(0)=1$.)

Note that the steady-state solution of the problem when $t \rightarrow \infty$ is $C(r, \infty)=M^{0} / V_{c}$, where $V_{c}=4 \pi r_{2}^{3} / 3$ is the volume of the container. Therefore, we have, from (2.10), 


$$
A_{0}=M^{0} / V_{c}
$$

Before determining the coefficients $A_{n}, n=1,2, \ldots$, we first note that

$$
\int_{0}^{r_{2}} j_{0}\left(\frac{r \alpha_{m}}{r_{2}}\right) \cdot j_{0}\left(\frac{r \alpha_{n}}{r_{2}}\right) r^{2} d r=\left\{\begin{array}{cc}
0, & m \neq n, \alpha_{m}, \alpha_{n} \geq 0 \\
\frac{r_{2}^{3}}{2} \cos ^{2} \alpha_{n}, & m=n ; \alpha_{n}>0 .
\end{array}\right.
$$

The derivation of this integral is given in the appendix.

We now use the initial condition (2.3) and the above results to determine $A_{n}$, for $n=1,2, \ldots$. Applying the initial condition (2.3) to (2.10) and using (2.11) we have

$$
C(r, 0)=\frac{M^{0}}{V_{c}}+\sum_{n=1}^{\infty} A_{n} j_{0}\left(\frac{r \alpha_{n}}{r_{2}}\right)=H(r)
$$

where $H$ is the function defined in (2.4). Multiplying both sides of the above equation by $r^{2} j_{0}\left(r \alpha_{m} / r_{2}\right)$ for any $m=1,2, \ldots$, integrating the resulting equation from 0 to $r_{2}$ and using (2.4), where $H(r)$ is zero from $r_{1}$ to $r_{2}$, and (2.12), we have

$$
\begin{aligned}
A_{m} \cdot \frac{r_{2}^{3}}{2} \cos ^{2} \alpha_{m} & =\frac{3 M^{0}}{4 \pi r_{1}^{3}} \int_{0}^{r_{1}} r^{2} j_{0}\left(r \frac{\alpha_{m}}{r_{2}}\right) d r \\
& =\frac{3 M^{0}}{4 \pi r_{1}^{3}} \int_{0}^{\sigma \alpha_{m}} \frac{r_{2}^{3}}{\alpha_{m}^{3}} u \sin u d u \quad\left(u=r \alpha_{m} / r_{2}, \sigma=r_{1} / r_{2}\right) \\
& =\frac{3 M^{0}}{4 \pi r_{1}^{3}} \frac{r_{2}^{3}}{\alpha_{m}^{3}}[\sin u-u \cos u]_{0}^{\sigma \alpha_{m}} \\
& =\frac{3 M^{0}}{4 \pi r_{1}^{3}} \frac{r_{2}^{3}}{\alpha_{m}^{3}}\left[\sin \left(\sigma \alpha_{m}\right)-\sigma \alpha_{m} \cos \left(\sigma \alpha_{m}\right)\right] \\
& =\frac{3 M^{0}}{4 \pi r_{1}^{3}} \frac{r_{2}^{3}}{\alpha_{m}^{3}}\left(\sigma \alpha_{m}\right)^{2} j_{1}\left(\sigma \alpha_{m}\right) \\
& =\frac{3 M^{0}}{4 \pi \sigma \alpha_{m}} j_{1}\left(\sigma \alpha_{m}\right)
\end{aligned}
$$


where $j_{1}(z)=(\sin z) / z^{2}-(\cos z) / z$ is the $1^{\text {st }}$ order spherical Bessel function (cf., for example, [25], p233). We thus have

$$
A_{m}=\frac{3 M^{0}}{2 \pi \sigma \alpha_{m} r_{2}^{3} \cos ^{2} \alpha_{m}} j_{1}\left(\sigma \alpha_{m}\right), m=1,2, \ldots
$$

Substituting (2.11) and (2.13) into (2.10) we finally get

$$
C(r, t)=\frac{M^{0}}{V_{c}}+\frac{3 M^{0}}{2 \pi \sigma r_{2}^{3}} \sum_{n=1}^{\infty} \frac{j_{1}\left(\sigma \alpha_{n}\right)}{\alpha_{n} \cos ^{2} \alpha_{n}} j_{0}\left(\frac{\alpha_{n} r}{r_{2}}\right) e^{-D \alpha_{n}^{2} t / r_{2}^{2}}
$$

This is an analytical solution to (2.1)-(2.3) in the region defined by $0<r<r_{2}$ and $0<t<\infty$.

\subsection{Total mass released in $[0, t]$ from the device}

We now derive the total mass released from the device into the container in the time interval $[0, t]$, denoted as $M_{t}$. For clarity, we let

$$
K_{n}(t)=\frac{j_{1}\left(\sigma \alpha_{n}\right)}{\alpha_{n} \cos ^{2} \alpha_{n}} e^{-D \alpha_{n}^{2} t / r_{2}^{2}}
$$

Multiplying both sides of (2.14) by $r^{2} \sin \phi \cdot d \theta \cdot d \phi \cdot d r$ and integrating the resulting equation over the region: $0 \leq \theta \leq 2 \pi, 0 \leq \phi \leq \pi$ and $r_{1} \leq r \leq r_{2}$, we have

$$
\begin{aligned}
M_{t} & =4 \pi \int_{r_{1}}^{r_{2}} C(r, t) r^{2} d r \\
& =\frac{M^{0}\left(V_{c}-V_{d}\right)}{V_{c}}+\frac{6 M^{0}}{\sigma r_{2}^{3}} \sum_{n=1}^{\infty} K_{n}(t) \int_{r_{1}}^{r_{2}} j_{0}\left(\frac{\alpha_{n} r}{r_{2}}\right) r^{2} d r \\
& =\frac{M^{0}\left(V_{c}-V_{d}\right)}{V_{c}}+\frac{6 M^{0}}{\sigma r_{2}^{3}} \sum_{n=1}^{\infty} K_{n}(t) \int_{\sigma \alpha_{n}}^{\alpha_{n}} \frac{r_{2}^{3}}{\alpha_{n}^{3}} u \sin u d u \quad\left(u=\frac{r \alpha_{m}}{r_{2}}, \sigma=\frac{r_{1}}{r_{2}}\right) \\
& =\frac{M^{0}\left(V_{c}-V_{d}\right)}{V_{c}}+\frac{6 M^{0}}{\sigma r_{2}^{3}} \sum_{n=1}^{\infty} K_{n}(t) \frac{r_{2}^{3}}{\alpha_{n}^{3}}[\sin u-u \cos u]_{\sigma \alpha_{n}}^{\alpha_{n}} \\
& =\frac{M^{0}\left(V_{c}-V_{d}\right)}{V_{c}}-6 M^{0} \sigma \sum_{n=1}^{\infty} \frac{K_{n}(t)}{\alpha_{n}} j_{1}\left(\sigma \alpha_{n}\right)
\end{aligned}
$$




$$
=\frac{M^{0}\left(V_{c}-V_{d}\right)}{V_{c}}-6 M^{0} \sigma \sum_{n=1}^{\infty} \frac{j_{1}^{2}\left(\sigma \alpha_{n}\right)}{\alpha_{n}^{2} \cos ^{2} \alpha_{n}} e^{-D \alpha_{n}^{2} t / r_{2}^{2}} .
$$

In the above we used (2.8). When $t \rightarrow \infty$, we have

$$
M_{t} \quad \rightarrow \quad M_{\infty}=\frac{M^{0}}{V_{c}}\left(V_{c}-V_{d}\right)
$$

which is the total mass released from the device into the external volume in the time interval $[0, \infty]$. Dividing both sides of $(2.15)$ by $M_{\infty}$ gives

$$
\frac{M_{t}}{M_{\infty}}=1-\frac{6 \sigma}{1-\sigma^{3}} \sum_{n=1}^{\infty} \frac{j_{1}^{2}\left(\sigma \alpha_{n}\right)}{\alpha_{n}^{2} \cos ^{2} \alpha_{n}} e^{-D \alpha_{n}^{2} t / r_{2}^{2}}=1-\frac{6 \sigma}{1-\sigma^{3}} \sum_{n=1}^{\infty} \frac{j_{1}^{2}\left(\sigma \alpha_{n}\right)}{\sin ^{2} \alpha_{n}} e^{-D \alpha_{n}^{2} t / r_{2}^{2}}
$$

This is a formula for the ratio of the mass released from the device into the liquid during the time interval $[0, t]$ and the total mass release from the device in infinite time. We comment that the deduction of (2.16) is based on the assumptions that the device and container are concentric as depicted in Figure 2 and that the diffusion in the liquid is homogeneous. These assumptions are normally satisfied in ideal laboratory conditions. When the assumptions are not satisfied, the diffusion problem (2.1)-(2.3) can only be solved by a full numerical method which will be discussed in a future paper.

\subsection{The initial burst}

A burst often appears in the initial phase of a release process. This is because, during the drug load process, some free drugs are left on the device surface. In this case, the initial release rate is substantially greater than that during the rest of the process. On the other hand, the initial release rate may also be much smaller than the normal rate if a device is pre-washed to remove the free drugs on the device surface. In both cases, it is desirable to identify the initial burst and its effect on the diffusion process. To characterize the initial burst, we assume that the effective diffusion coefficient is a piecewise constant in time, i.e., 


$$
D=\left\{\begin{array}{lc}
D_{0}, & 0<t<t_{c} \\
D_{1}, & t>t_{c}
\end{array}\right.
$$

where $D_{0}$ and $D_{1}$ are constants and $t_{c}$ is the threshold time. All of these parameters are yet to be determined. From Section 2.2 we see that when $0 \leq t \leq t_{c}$, the concentration $C(r, t)$ is given by (2.14) with $D=D_{0}$. Using the same argument as that for (2.10) we have

$$
C(r, t)=\sum_{n=0}^{\infty} \bar{A}_{n} j_{0}\left(\frac{\alpha_{n} r}{r_{2}}\right) e^{-D_{1} \alpha_{n}^{2} t / r_{2}^{2}}, \quad t>t_{c}
$$

where $\bar{A}_{n}$ 's are coefficients to be determined. Using the same argument employed for determining $A_{0}$ in (2.10) we have $\bar{A}_{0}=M^{0} / V_{c}$. The continuity condition at $t_{c}$ that $C\left(r, t_{c}^{-}\right)=C\left(r, t_{c}^{+}\right)$for all admissible $r$ gives

$$
\sum_{n=0}^{\infty} \bar{A}_{n} j_{0}\left(\frac{\alpha_{n} r}{r_{2}}\right) e^{-D_{1} \alpha_{n}^{2} t_{c} / r_{2}^{2}}=A_{0}+\frac{3 M^{0}}{2 \pi \sigma \sigma_{2}^{3}} \sum_{n=1}^{\infty} \frac{j_{1}\left(\sigma \alpha_{n}\right)}{\alpha_{n} \cos ^{2} \alpha_{n}} j_{0}\left(\frac{\alpha_{n} r}{r_{2}}\right) e^{-D_{0} \alpha_{n}^{2} t_{c} / r_{2}^{2}}
$$

Matching the coefficients on both sides of the above equality, we have

$$
A_{0}=\bar{A}_{0}=\frac{M^{0}}{V_{c}} \text { and } \bar{A}_{n}=\frac{3 M^{0}}{2 \pi \sigma r_{2}^{3}} \frac{j_{1}\left(\sigma \alpha_{n}\right)}{\alpha_{n} \cos ^{2} \alpha_{n}} e^{-\left(D_{0}-D_{1}\right) \alpha_{n}^{2} t_{c} / r_{2}^{2}}
$$

for $n \geq 1$. Combining this with (2.17) we have the expression for $C(r, t)$ when $t>t_{c}$.

It is clear that when $0 \leq t \leq t_{c}, \frac{M_{t}}{M_{\infty}}$ is given by (2.16) with $D=D_{0}$. Using the same argument as that for (2.16), it is easy to show from (2.17) and (2.18) that

$$
\frac{M_{t}}{M_{\infty}}=1-\frac{6 \sigma}{\left(1-\sigma^{3}\right)} \sum_{n=1}^{\infty} \frac{j_{1}^{2}\left(\sigma \alpha_{n}\right)}{\sin ^{2} \alpha_{n}} e^{-\alpha_{n}^{2}\left(D_{1}\left(t-t_{c}\right)+D_{0} t_{c}\right) / r_{2}^{2}}, \text { for } t>t_{c} .
$$

\subsection{Effective boundary layer}


When the liquid is well stirred, the concentration can be considered uniform in most of the liquid region except for a thin layer, called the boundary layer, around the device. In this case, the diffusion dominates the mass transfer only in the boundary layer region. For simplicity we assume that thickness of the boundary layer is uniform around the device. Let $r_{1}$ denote the radius of the device, $r_{2}-r_{1}$ the thickness of the boundary layer and $r_{3}$ the radius of the container, satisfying $0<r_{1}<r_{2} \leq r_{3}$. The geometry is depicted in Figure 3 .

Figure 3: A spherical device with radius $r_{1}$ placed in a container with radius $r_{3}$.

The problem can be formulated as

$$
\begin{aligned}
& \frac{\partial \hat{C}(r, t)}{\partial t}=D\left(\frac{\partial^{2} \hat{C}(r, t)}{\partial r^{2}}+\frac{2}{r} \frac{\partial \hat{C}(r, t)}{\partial r}\right), \quad 0<r<r_{2}, \quad t>0, \\
& \hat{C}(r, t)=C_{0}(t), \quad r_{2} \leq r \leq r_{3}, \quad t>0, \\
& \hat{C}(r, 0)=\left\{\begin{array}{cl}
\frac{M^{0}}{V_{d}}, & 0<r \leq r_{1}, \\
0, & r_{1}<r<r_{3},
\end{array}\right.
\end{aligned}
$$

where $V_{d}=4 \pi r_{1}^{3} / 3$, the volume of the device as defined before, and $C_{0}(t)$ is the (unknown) concentration outside the layer. The second equation above represents the fact that from $r_{2}$ 
to $r_{3}$, the concentration is uniform. Using the results in Section 2.1 it is easy to verify that the solution to this problem is

$$
\hat{C}(r, t)=\left\{\begin{array}{cc}
C(r, t), & r \in\left(0, r_{2}\right), \\
C_{0}(t) & r \in\left[r_{2}, r_{3}\right]
\end{array}\right.
$$

for $t>0$ with the continuity condition $C_{0}(t)=C\left(r_{2}, t\right)$, where $C(r, t)$ is given by (2.14). We now calculate the total mass, $M_{t}$, released in time $t$. Note that $M_{t}$ contains two parts: the mass at $t$ in the boundary layer region $\left(r_{1}, r_{2}\right) \times(0,2 \pi)$ and that in the convection-dominant region $\left(r_{2}, r_{3}\right) \times(0,2 \pi)$. The calculation of the former is exactly the same as that for (2.16) and the latter is just the constant concentration $C\left(r_{2}, t\right)$ times the corresponding volume.

Let $V_{c}=4 \pi r_{2}^{3} / 3$, as defined before, and $\hat{V}_{c}=4 \pi r_{3}^{3} / 3$, the volume of the container. Following the derivation of (2.16) we have, from (2.14), (2.20) and the above analysis,

$$
\begin{aligned}
\hat{M}_{t}= & \frac{M^{0}}{\hat{V}_{c}}\left(V_{c}-V_{d}\right)-6 M^{0} \sigma \sum_{n=1}^{\infty} \frac{j_{1}^{2}\left(\sigma \alpha_{n}\right)}{\sin ^{2} \alpha_{n}} e^{-D \alpha_{n}^{2} t / r_{2}^{2}}+C\left(r_{2}, t\right)\left(\hat{V}_{c}-V_{c}\right) \\
= & \frac{M^{0}}{\hat{V}_{c}}\left(\hat{V}_{c}-V_{d}\right)-6 M^{0} \sigma \sum_{n=1}^{\infty} \frac{j_{1}^{2}\left(\sigma \alpha_{n}\right)}{\sin ^{2} \alpha_{n}} e^{-D \alpha_{n}^{2} t / r_{2}^{2}} \\
& +\frac{3 M^{0}}{2 \pi \sigma r_{2}^{3}}\left(\hat{V}_{c}-V_{c}\right) \sum_{n=1}^{\infty} \frac{j_{1}\left(\sigma \alpha_{n}\right)}{\alpha_{n} \cos ^{2} \alpha_{n}} j_{0}\left(\alpha_{n}\right) e^{-D \alpha_{n}^{2} t / r_{2}^{2}}
\end{aligned}
$$

Let $\hat{M}_{\infty}=\frac{M^{0}}{\hat{V}_{c}}\left(\hat{V}_{c}-V_{d}\right)$, representing the total mass in the liquid after infinite time. Dividing both sides of the above equation by $\hat{M}_{\infty}$, we have

$$
\begin{aligned}
& \frac{\hat{M}_{t}}{\hat{M}_{\infty}}=1-\frac{6 \sigma}{\left(1-\rho^{3}\right)} \sum_{n=1}^{\infty} \frac{j_{1}^{2}\left(\sigma \alpha_{n}\right)}{\sin ^{2} \alpha_{n}} e^{-D \alpha_{n}^{2} t / r_{2}^{2}}+\frac{2\left(\frac{1}{\gamma^{3}}-1\right)}{\sigma\left(1-\rho^{3}\right)} \sum_{n=1}^{\infty} \frac{\alpha_{n} j_{1}\left(\sigma \alpha_{n}\right)}{\sin ^{2} \alpha_{n}} j_{0}\left(\alpha_{n}\right) e^{-D \alpha_{n}^{2} t / r_{2}^{2}} \\
& =1-\frac{2}{\left(1-\rho^{3}\right)} \sum_{n=1}^{\infty} \frac{j_{1}\left(\sigma \alpha_{n}\right)}{\sin ^{2} \alpha_{n}}\left(3 \sigma j_{1}\left(\sigma \alpha_{n}\right)-\frac{\alpha_{n} j_{0}\left(\alpha_{n}\right)}{\sigma}\left(\frac{1}{\gamma^{3}}-1\right)\right) e^{-D \alpha_{n}^{2} t / r_{2}^{2}}
\end{aligned}
$$


Where $\sigma=\frac{r_{1}}{r_{2}}, \gamma=\frac{r_{2}}{r_{3}}$ and $\rho=\frac{r_{1}}{r_{3}}$.

Using (2.21) and the technique in Section 2.3 for deducing (2.19) it is easy to derive the following formula containing both the initial burst and the convection phenomena: i.e., $\frac{\hat{M}_{t}}{\hat{M}_{\infty}}$ is given by (2.21) with $D=D_{0}$ when $0<t \leq t_{c}$ and

$$
\frac{\hat{M}_{t}}{\hat{M}_{\infty}}=1-\frac{2}{\left(1-\rho^{3}\right)} \sum_{n=1}^{\infty} \frac{j_{1}\left(\sigma \alpha_{n}\right)}{\sin ^{2} \alpha_{n}}\left(3 \sigma j_{1}\left(\sigma \alpha_{n}\right)-\frac{\alpha_{n} j_{0}\left(\alpha_{n}\right)}{\sigma}\left(\frac{1}{\gamma^{3}}-1\right)\right) e^{-\alpha_{n}^{2}\left(D_{1}\left(t-t_{c}\right)+D_{0} t_{c}\right) / r_{2}^{2}}
$$

for $t>t_{c}$, where $t_{c}$ is the effective critical time.

We comment that the thickness of the boundary layer, $r_{2}-r_{1}$, can not normally be determined exactly. In this investigation, we treat $r_{2}$ as a decision parameter in an optimization process, and refer to the resulting value as the effective boundary layer.

\section{Device preparation, drug loading and release experiments}

\subsection{Chemicals and materials}

HEMA (Bimax, ophthalmic grade) was used as received. The cross-linking agent 1,5hexadiene-3,4-diol (DVG) with a purity of $97 \%$ was supplied by Sigma-Aldrich. An aqueous solution of $10 \mathrm{wt} \%$ ammonium persulphate (APS) (Ajax Chemicals) was used together with N,N,N',N'-tetramethylethylene diamine (TEMED) (Aldrich Chemical Co.) as initiators. Prednisolone 21-hemissucinate sodium salt powder was purchased from Sigma Chemical Co., Belgium. Deionised water was used for all experiments in the study. 


\subsection{Device preparation}

Three porous PHEMA sphercal devices, S2080, S3070 and S4060 were cast following the specifications given in Table 1. The formulations were selected to produce devices chemically identical but structurally different. The physical properties including the swelling behaviour, the polymer volume fraction, density of the dry and wet polymer hydrogels have been reported in our previous work $[11,12]$. To cast the polymer devices, HEMA and water were well mixed in a beaker followed by the addition of the cross-linking agent (DVG) and the initiators (APS and TEMED). The solution was then distributed into a plastic mold as displayed in Figure 4a. Polymerization was carried out at room temperature for 3 hours and then at $50^{\circ} \mathrm{C}$ for 24 hours. Following the polymerisation, the samples were removed from the mould and immersed in deionised water for 4 weeks with daily water exchange to remove residual monomers and oligomers. Photographs of td the produced spherical devices are displayed in Figure 4b.

Table 1. Chemical formulations for PHEMA device preparation

\begin{tabular}{|c|c|c|c|c|c|}
\hline Device & HEMA & Water & DVG & APS (10\%) & TEMED \\
Name & $(\mathbf{g})$ & $(\mathbf{g})$ & $(\boldsymbol{\mu L})$ & $(\boldsymbol{\mu L})$ & $(\boldsymbol{\mu L})$ \\
\hline $\mathbf{S 2 0 8 0}$ & 50 & 200 & 500 & 1000 & 1000 \\
\hline $\mathbf{S 3 0 7 0}$ & 75 & 175 & 750 & 1500 & 1500 \\
\hline $\mathbf{S 4 0 6 0}$ & 100 & 150 & 1000 & 2000 & 2000 \\
\hline
\end{tabular}


Figure 4. Photographs of (a) the plastic mould and (b) the spherical PHEMA devices.

\subsection{Drug loading and release experiments}

Upon completion of water exchange the devices were freeze-dried and placed in containers containing a drug solution of either $1.0 \mathrm{wt} \%$ or $0.5 \mathrm{wt} \%$ concentration. Sufficient drug solution was added to allow the maximum absorption of the drugs upon swelling of the devices.

The drug loaded devices, S2080-10, S3070-10, S40-60-10, S2080-05, S3070-05 and S406005 were then placed in the centre of a container which has an air tight seal (10 and 05 are used in the sample codes representing $1.0 \mathrm{wt} \%$ and $0.5 \mathrm{wt} \%$ drug solution respectively). The container was then filled with enough deionised water and placed upon an orbital shaker (Chiltern Scientific) at a speed of $45 \mathrm{rpm}$. At preset time points $500 \mu \mathrm{L}$ of the drug solution was removed from a marked location and further diluted for quantitative analysis of released drug concentrations $M_{t}$ using a UV-Vis spectrometer. Details of the quantitative analysis of drugs can be found in references $[11,12]$.

\section{Results and discussions}

\subsection{Testing the mathematical models}

In this section we will test the models established in the previous section using some experimental data. 
The series solutions obtained from Section 3 contain up to four unknown parameters $D_{0}, D_{1}, t_{c}$ and $r_{2}$. To determine these parameters, a nonlinear least-squares algorithm is used as proposed in [23]. The algorithm is to minimize the fitting error

$$
E\left(t_{c}, D_{0}, D_{1}, \theta\right)=\sum_{k=1}^{K}\left(R_{e}\left(t_{k}\right)-R_{N}\left(t_{k}, t_{c}, D_{0}, D_{1}, \theta\right)\right)^{2} w_{k},
$$

where $w_{k}$ is a positive constant, $R_{e}\left(t_{k}\right)$ is the experimentally measured value of the ratio $\frac{M_{t}}{M_{\infty}}\left(\right.$ or $\left.\frac{\hat{M}_{t}}{\hat{M}_{\infty}}\right)$ at $t_{k}$ for $k=1,2, \ldots, K$, and $\theta=\left(r_{2}-r_{1}\right) /\left(r_{3}-r_{1}\right)$ is a parameter characterizing the width of the boundary layer satisfying $0<\theta \leq 1$. The quantity $R_{N}$ in (4.1) is the sum of the first $N$ terms of an exact solution of the ratio. For instance, $R_{N}$ for the solution in Section 2.3 (i.e., (2.16) and (2.19)) is given by

$$
R_{N}\left(t, t_{c}, D_{0}, D_{1}\right)=\left\{\begin{array}{cc}
1-\frac{6 \sigma}{\left(1-\sigma^{3}\right)} \sum_{n=1}^{N} \frac{j_{1}^{2}\left(\sigma \alpha_{n}\right)}{\sin ^{2} \alpha_{n}} e^{-D_{0} \alpha_{n}^{2} t / r_{2}^{2}}, & t \leq t_{c} \\
1-\frac{6 \sigma}{\left(1-\sigma^{3}\right)} \sum_{n=1}^{N} \frac{j_{1}^{2}\left(\sigma \alpha_{n}\right)}{\sin ^{2} \alpha_{n}} e^{-\alpha_{n}^{2}\left(D_{1}\left(t-t_{c}\right)+D_{0} t_{c}\right) / r_{2}^{2}}, & t>t_{c}
\end{array}\right.
$$

(In this case $\theta=1$ is not a decision variable since $r_{2}=r_{3}$.) For simplicity, we assume that $t_{c}$ only takes values from the discrete set $\left\{t_{1}, t_{2}, \ldots, t_{K}\right\}$. For all the tests below, we choose $N=62$. The first 62 roots of (2.8) are calculated numerically using Matlab. To avoid possible local minima, the least-squares problem is solved using the following initial starting points

$$
D_{0}=D_{1}=10^{-5} / 2^{i} \text { for } i=1,2, \ldots, 10 \text { and } \theta=0.1 j \text { for } j=1,2, \ldots, 10
$$

The weights in (4.1) are chosen to be $w_{k}=K\left(t_{k}-t_{k-1}\right) / t_{N}$ for $k=1,2, \ldots, K$ with $t_{0}=0$. 
In what follows, we shall refer the solution (2.16) to as Model BM, the solution (2.1) and (2.19) to as Model IB, (2.21) to as Model BL and (2.21) and (2.22) to as Model IB+BL.

\subsubsection{The effect of initial burst}

In order to see the effect of initial burst, experimental data of S2080-10, $M_{t} / M_{\infty}$, collected at 12 time points from 0.5 to 72.8 (Table 2) were fitted with all four models. The radius of the device, $r_{1}$, was measured as $0.906 \mathrm{~cm}$ and the radius of the effective container, i.e., $r_{2}$ in Models BM and IB or $r_{3}$ in Models BL and IB+BL, used in experiments is $2.037 \mathrm{~cm}$.

Table 2: Experimental data of $M_{t} / M_{\infty}$ for S2080-10 and S4060-05

\begin{tabular}{|l|l|l|l|l|l|l|}
\hline Time (hour) & $\mathbf{0 . 5}$ & $\mathbf{1 . 0}$ & $\mathbf{1 . 5}$ & $\mathbf{2 . 0}$ & $\mathbf{3 . 0}$ & $\mathbf{4 . 5}$ \\
\hline S2080-10 & 0.577 & 0.671 & 0.675 & 0.686 & 0.697 & 0.732 \\
\hline S4060-05 & 0.158 & 0.212 & 0.230 & 0.260 & 0.286 & 0.332 \\
\hline Time (hours) & $\mathbf{6 . 9}$ & $\mathbf{2 4 . 9}$ & $\mathbf{3 2 . 7}$ & $\mathbf{5 1 . 0}$ & $\mathbf{5 5 . 8}$ & $\mathbf{7 2 . 8}$ \\
\hline S2080-10 & 0.866 & 0.934 & 0.963 & 0.989 & 0.980 & 1.000 \\
\hline S4060-05 & 0.365 & 0.645 & 0.781 & 0.971 & 0.973 & 1.000 \\
\hline
\end{tabular}

The fitted curves using the four models BM, IB, BL and IB+BL are displayed in Figure 5. The curves fitted by BM and BL are almost identical, so are those fitted by IB and IB+BL which indicate an insignificant boundary layer effect for the selected device. On the other hand, an apparent initial burst from device S2080-10 is identified by both IB and IB+BL. The fittings by Models IB and IB+BL are more satisfactory than those by Models BM and BL. The more adequate approximation by Models IB and IB+BL is also demonstrated by a tenfold smaller value of the lease squares error of these methods in comparison with the other two models (Table 3). 


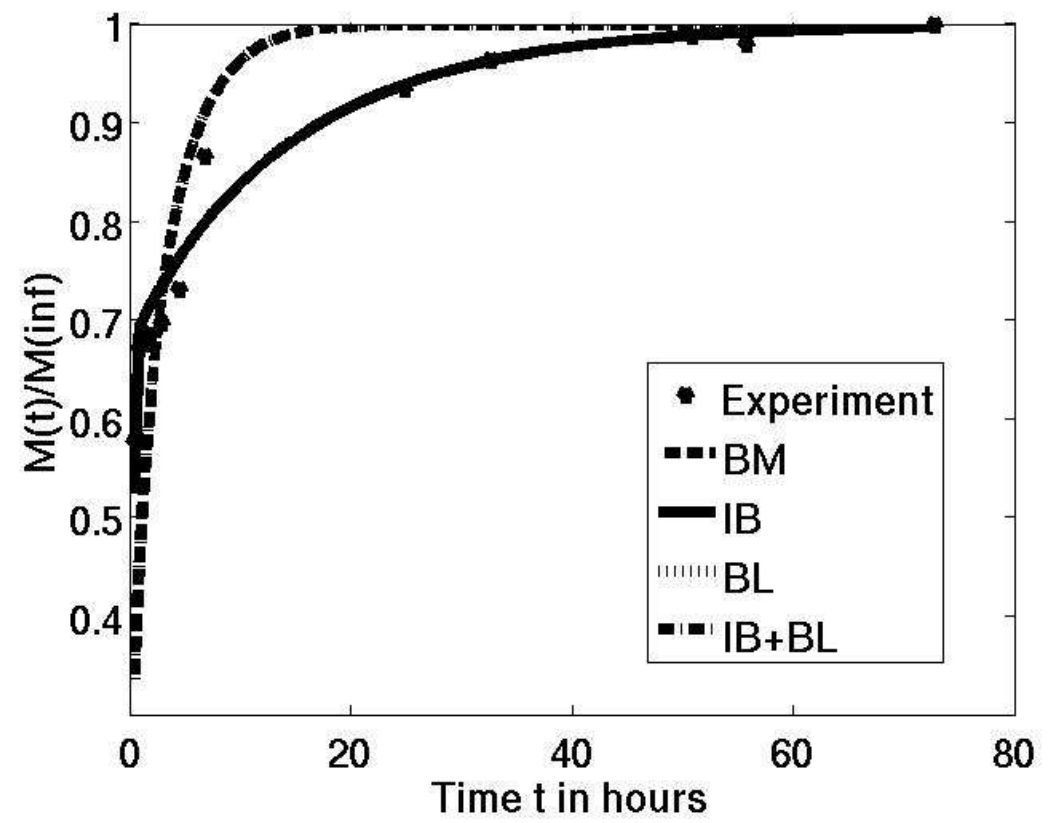

Figure 5: Fitted curves by the four mathematical models for S2080-10.

Table 3: Results from Models BM, IB, BL and IB+BL for S2080-10

\begin{tabular}{|l|l|l|l|l|}
\hline Model & Diffusivity $\left(\mathrm{cm}^{2} / \mathrm{s}\right)$ & $t_{\mathrm{c}}$ (hour) & $\theta$ & Least-squares Error \\
\hline BM & $1.56 \mathrm{E}-5$ & - & - & $2.81 \mathrm{E}-2$ \\
\hline IB & $(4.13 \mathrm{E}-5,3.65 \mathrm{E}-6)$ & 1.0 & - & $2.85 \mathrm{E}-3$ \\
\hline BL & $1.56 \mathrm{E}-5$ & - & 1.00 & $2.81 \mathrm{E}-2$ \\
\hline IB+BL & $(4.13 \mathrm{E}-5,3.65 \mathrm{E}-6)$ & 1.0 & 1.00 & $2.85 \mathrm{E}-3$ \\
\hline
\end{tabular}

\subsubsection{The effective boundary layer}

To determine the effective boundary layer, similar fittings were performed on the experimental data from the device S4060-05 (Table 2). The fitted curves from the four models are displayed in Figure 6 and the computed optimal parameters are listed in Table 4. In this case, a minor drug burst during the first two hours is revealed by both IB and IB+BL (Fig. 6). In addition, effective boundary layers, measured as $\theta=93 \%$ and $84 \%$, are identified 
by $\mathrm{BL}$ and $\mathrm{IB}+\mathrm{BL}$ respectively (Table 4 ). For this particular device, the computed values of the effective diffusion coefficient from the four models are similar. However, the best fit is obtained by the combined model $\mathrm{IB}+\mathrm{BL}$ which is demonstrated by the smallest least squares error of the fitting.

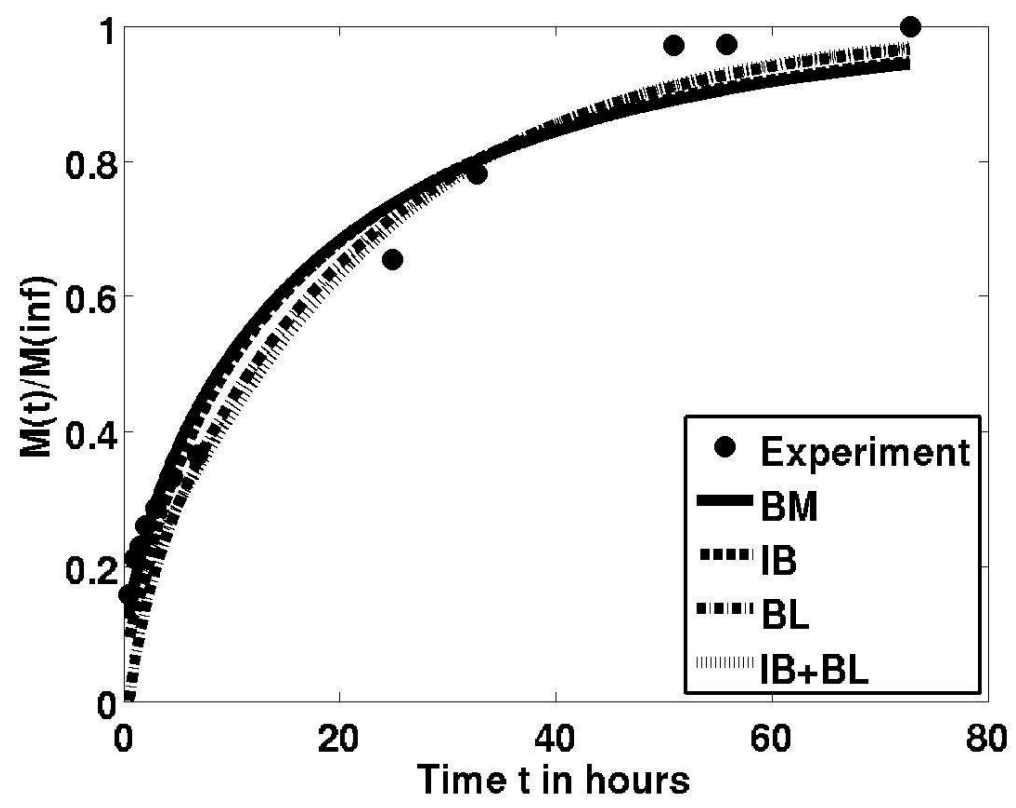

Figure 6: Fitted curves by the four mathematical models for S4060-05.

Table 4: Results from Models BM, IB, BL and IB+BL for S4060-05

\begin{tabular}{|l|l|l|l|l|}
\hline Model & Diffusivity $\left(\mathrm{cm}^{2} / \mathrm{s}\right)$ & $t_{\mathrm{c}}$ (hour) & $\theta$ & Least-squares Error \\
\hline BM & $1.55 \mathrm{E}-6$ & - & - & $5.51 \mathrm{E}-2$ \\
\hline IB & $(1.25 \mathrm{E}-6,1.86 \mathrm{E}-6)$ & 2.0 & - & $5.28 \mathrm{E}-2$ \\
\hline BL & $2.10 \mathrm{E}-6$ & - & 0.93 & $3.69 \mathrm{E}-2$ \\
\hline IB+BL & $(2.94 \mathrm{E}-5,2.00 \mathrm{E}-6)$ & 0.5 & 0.84 & $2.44 \mathrm{E}-2$ \\
\hline
\end{tabular}

\subsection{Determination of the diffusion parameters}

Applying the four mathematical models to the experimental data of all investigated devices 
has indicated that, 1) models IB and IB+BL yield better fitting and approximation results when an initial burst of drugs occurs, 2) the effective boundary layers are not always present in the spherical devices investigated in this study, however when the effect is apparent, models $\mathrm{BL}$ and $\mathrm{IB}+\mathrm{BL}$ are more efficient to identify the phenomenon, and 3) in all devices, the combined model $\mathrm{IB}+\mathrm{BL}$ has produced more satisfactory results than the individual models developed, judged by both the ability to identify the effect of the initial burst and the effective boundary layers, as well as by the smaller least square errors. Therefore only the computed parameters from Model IB+BL are listed and used for further discussions (Table 5). Fittings of all experimental data by the combined model are shown in Figure $7 \mathrm{a}$ and $7 \mathrm{~b}$.

Table 5: Computed diffusion parameters of all devices by Model IB+BL

\begin{tabular}{|c|c|c|c|c|c|}
\hline \multirow{2}{*}{ Device } & \multicolumn{2}{|l|}{ Diffusion coefficient $\left(\mathrm{cm}^{2} / s\right)$} & \multirow{2}{*}{$\theta$} & \multirow{2}{*}{$t_{c}$ (hour) } & Least-squares Error \\
\cline { 2 - 4 } & $\mathrm{D}_{0}$ & $\mathrm{D}_{1}$ & & & \\
\hline S2080-10 & $4.13 \mathrm{E}-05$ & $3.65 \mathrm{E}-06$ & 1.00 & 1.0 & $2.85 \mathrm{E}-03$ \\
\hline S3070-10 & $5.03 \mathrm{E}-06$ & $2.30 \mathrm{E}-06$ & 1.00 & 1.0 & $5.04 \mathrm{E}-03$ \\
\hline S4060-10 & $1.49 \mathrm{E}-05$ & $2.20 \mathrm{E}-06$ & 0.92 & 1.0 & $5.11 \mathrm{E}-03$ \\
\hline S2080-05 & $6.01 \mathrm{E}-06$ & $2.83 \mathrm{E}-06$ & 0.98 & 1.0 & $7.67 \mathrm{E}-03$ \\
\hline S3070-05 & $3.44 \mathrm{E}-06$ & $2.02 \mathrm{E}-06$ & 0.94 & 2.0 & $8.35 \mathrm{E}-03$ \\
\hline S4060-05 & $2.94 \mathrm{E}-05$ & $2.00 \mathrm{E}-06$ & 0.84 & 0.5 & $2.44 \mathrm{E}-02$ \\
\hline
\end{tabular}

\subsection{Comparison of the diffusion parameters}

The computed parameters listed in Table 5 demonstrate that the corrected effective diffusion coefficient, $D_{1}$, of S2080 is greater than that of S3070, and greater still than that of S4060 at both drug loading levels. The descending trend of $D_{1}$ in devices loaded with $1.0 \mathrm{wt} \%$ drug solutions is more significant than that in devices loaded with $0.5 \mathrm{wt} \%$ drug solutions. These 
observations are coincide with the fact that S2080 has a more porous structure than the other two devices and are also in agreement with our previous results on the disc geometry [11, 12]. We have also noticed that the initial burst effect in $S 2080$ is more significant than in the other two devices (Table 3 and 5), indicating that the drugs are more prone to burst from S2080 due to its softer and more porous nature.

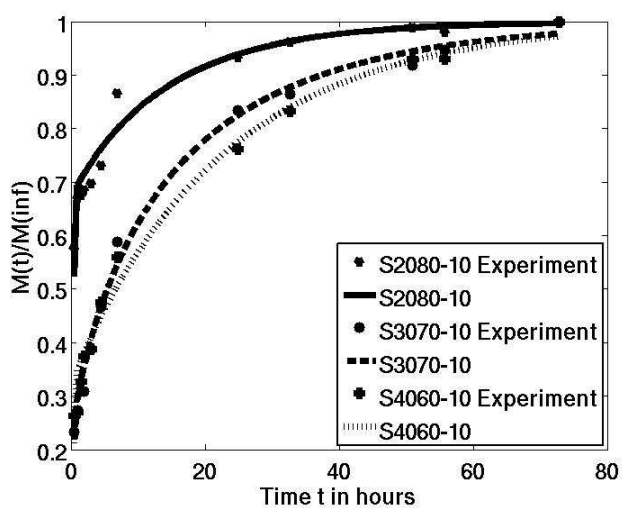

(a)

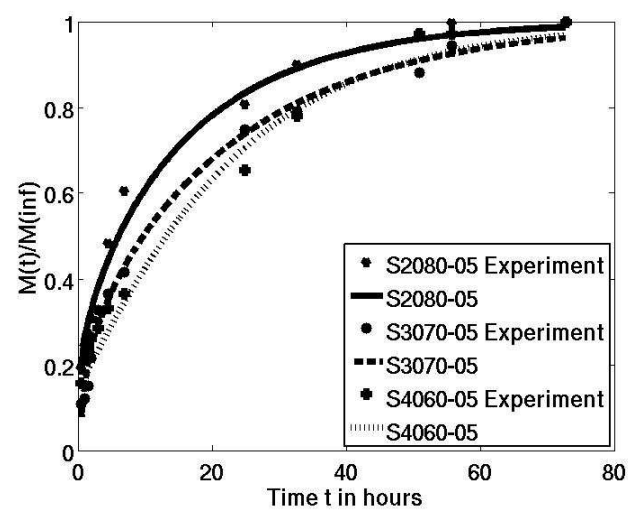

(b)

Figure 7: Fitted curves by Model IB+BL for devices loaded with (a) $1.0 \mathrm{wt} \%$ and (b) 0.5 wt\% drug solutions.

\section{Conclusions}

In this work we have developed a full mathematical model for extracting effective parameters such as diffusion coefficients, critical time of initial burst and width of boundary layers that determine the release process of a drug from a spherical device into a finite volume. The model contains three other simpler models as special cases. Explicit expressions for the analytical solutions of these models have been obtained which contain the parameters as unknown decision variables. A nonlinear least-squares method is then used for finding the optimal solutions to these parameters, yielding an optimal fit to a set of experimental data. Numerical experiments have been performed using laboratory observed data of three drug 
release devices made of porous hydrogel polymers with two different drug loading levels to show the accuracy and usefulness of the models. The results demonstrated that the full mathematical model can effectively identify both the drug burst effect and the effective boundary layer, if any, and therefore can more accurately determine the diffusion parameters that govern a true diffusion process, whilst the three simpler models are effective only for the uncontaminated experimental data. The computed diffusion parameters are explicable in terms of the drug loading concentrations and the porous structure of the devices and are generally consistent with the results obtained from our previous studies on the disc geometry. Full numerical methods such as those in references [26, 27] are under development for estimating effective diffusion parameters of drugs from hydrogel devices of more general geometries.

\section{Acknowledgements}

The authors are grateful to the Australian Research Council (ARC) for financial assistance.

\section{References}

[1] Siepmann J., Siepmann F. (2008) Mathematical modelling of drug delivery. International Journal of Pharmaceutics. 364:328-343.

[2] MacArthur B.D., Oreffo R.O.C. (2005) Bridging the gap. Nature, 436:19.

[3] Groves M.J. (1999) Parenteral drug delivery systems; in Encylopedia of Controlled Drug Delivery. Ed. E Mathoiwitz, New York: Wiley.

[4] Arora J.S. (1989) Introduction to optimum design, McGraw-Hill, New York.

[5] Hernández S. (1993) Advanced techniques in the optimum design of structures, Computational Mechanics Publications, Southampton-Boston.

[6] Wang S., Teo K.L., Lee H.W.J. (1998) A new approach to nonlinear mixed discrete 
programming problems, Engineering Optimization,30:249-262.

[7] Lee W.R., Wang S., Teo K.L. (1999) An optimization approach to a finite dimensional parameter estimation problem in semiconductor device design, $\mathrm{J}$. Comput. Phys., 156:241-256.

[8] Colin R. (1998) Mathematical modeling of controlled release from implanted drugimpregnated monoliths, PSTT, 1:269-276.

[9] Doumenc F. and Guerrier B.(2001) Estimating polymer/solvent diffusion coefficient by optimization procedure, AIChE J., 47:984-993.

[10] Lu S., Ramirez W.F. and Anseth K.S. (1998) Modelling and optimization of drug release from laminated polymer matrix devices. AIChE J., 44:1698-1696.

[11] Lou X., Munro S, Wang S. (2004) Drug release characteristics of phase separation PHEMA sponge materials. Biomaterials, 25:5071-5080.

[12] Lou X., Wang S., Tan S.Y. (2007) Mathematics-aided quantitative analysis of diffusion characteristics of PHEMA sponge hydrogels, Asia-Pac. J. Chem. Eng. 2:609-617.

[13] Pappas N.A. (Ed.) (1987) Hydrogels in Medicine and Pharmacy, Vol. II, CRC Press, Boca Taton.

[14] Refojo M.F. (1982) Polymers in Ophthalmology: An Overview in Biocompatibility in Clinical Practice Vol.II. Ed. D.F. Williams, CRC Press, Boca Raton, 3-18.

[15] Oxley H.R., Corkhill P.H., Fitton J.H. and Tighe B.H. (1993) Macroporous hydrogels for biomedical applications: Methodology and morphology., Biomaterials, 14:1064-1072.

[16] Crawford G.J., Hicks C.R., Lou X., Vijayasekaran S., Tan D., Chirila T.V., Constable I.J. (2002) The Chirila Keratoprosthesis: Phase I human clinical trials. Ophthalmology, 109:883-889. 
[17] Hicks C.R., Crawford G.J., Lou X., Tan T.D., et al. (2003) Cornea replacement using a synthetic hydrogel cornea, AlphaCor: device, preliminary outcomes and complications. Eye, 17:385-392.

[18] Hicks C.R., Morrison D., Lou X., Crawford G.J., Gadjatsy A,. Constable I.J. (2006) Orbit Implants: Potential new directions. Expert Rev Med Devices, 3:805-815.

[19] Grassi M., Grassi G. (2005) Mathematical modelling and controlled drug delivery: Matrix systems. Current Drug Delivery, 2:97-116.

[20] Fu J.C., Hagemeier C., Moyer D.L. (1976) A unified mathematical model for diffusion from drug-polyner composite tablets. J. Biomed Matter. Res., 10:743-758.

[21] Carslaw H.S., Jaeger J.C. (1959) Conduction of heat in solids. Oxford: Clarendon Press.

[22] Crank J. (1975) The mathematics of diffusion, 2nd ed. London: Oxford University Press.

[23] Wang S., Lou X. (2009) An optimization approach to the estimation of effective drug diffusivity: from a planar disc into a finite external volume. Journal of Industrial and Management Optimization, 5:127-140.

[24] Lou X., Vijayasekaran S., Sugiharti R., Robertson T. (2005) Morphological and topographic effect on calcification tendency of PHEMA hydrogels. Biomaterials, 26:5808-5817.

[25] Pinsky M.A. (1991) Partial differential equations and boundary value problems with applications. New York: McGraw-Hill.

[26] Zhou Y., Wu X.Y. (1997) Finite element analysis of diffusional drug release from complex matrix systems. I. Complex geometries and composite structures. J. Controlled Release, 49:277-288.

[27] Wang S., Lou X. (2009) Numerical methods for the estimation of effective 
diffusion coefficients of 2D controlled drug delivery systems, Optimization \& Engineering, in press, DOI: 10.1007/s11081-008-9069-8.

\section{Appendix : Derivation of (2.12)}

Case I: $m \neq n, \alpha_{m}, \alpha_{n}>0$

$$
\begin{aligned}
& \int_{0}^{r_{2}} j_{0}\left(\frac{r \alpha_{m}}{r_{2}}\right) \cdot j_{0}\left(\frac{r \alpha_{n}}{r_{2}}\right) r^{2} d r=\int_{0}^{r_{2}} \frac{r_{2} \sin \left(\frac{r \alpha_{m}}{r_{2}}\right)}{r \alpha_{m}} \cdot \frac{r_{2} \sin \left(\frac{r \alpha_{n}}{r_{2}}\right)}{r \alpha_{n}} r^{2} d r \\
& =\frac{r_{2}^{2}}{\alpha_{m} \alpha_{n}} \int_{0}^{r_{2}} \sin \left(\frac{r \alpha_{m}}{r_{2}}\right) \sin \left(\frac{r \alpha_{n}}{r_{2}}\right) d r \\
& =\frac{r_{2}^{2}}{\alpha_{m} \alpha_{n}}\left(-r_{2} \frac{\alpha_{m} \cos \left(\alpha_{m}\right) \sin \left(\alpha_{n}\right)-\alpha_{n} \sin \left(\alpha_{m}\right) \cos \left(\alpha_{n}\right)}{\left(\alpha_{m}-\alpha_{n}\right)\left(\alpha_{m}+\alpha_{n}\right)}\right) \\
& =\frac{-r_{2}^{3}}{\left(\alpha_{n} \alpha_{m}^{3}-\alpha_{m} \alpha_{n}^{3}\right)}\left[\alpha_{m} \cos \left(\alpha_{m}\right) \sin \left(\alpha_{n}\right)\right. \\
& \left.-\alpha_{n} \sin \left(\alpha_{m}\right) \cos \left(\alpha_{n}\right)-\sin \left(\alpha_{m}\right) \sin \left(\alpha_{n}\right)+\sin \left(\alpha_{m}\right) \sin \left(\alpha_{n}\right)\right] \\
& =\frac{-r_{2}^{3}}{\left(\alpha_{n} \alpha_{m}^{3}-\alpha_{m} \alpha_{n}^{3}\right)}\left[\sin \left(\alpha_{n}\right)\left(\alpha_{m} \cos \left(\alpha_{m}\right)-\sin \left(\alpha_{m}\right)\right)\right. \\
& -\sin \left(\alpha_{m}\right)\left(\alpha_{n} \cos \left(\alpha_{n}\right)-\sin \left(\alpha_{n}\right)\right] \\
& =0 \text {, }
\end{aligned}
$$

since $\alpha_{m}$ and $\alpha_{n}$ are roots of $\alpha \cos (\alpha)-\sin (\alpha)=0$.

Case II: $m=0, \alpha_{n}>0$,

$$
\begin{aligned}
& \int_{0}^{r_{2}} j_{0}(0) j_{0}\left(\frac{r \alpha_{n}}{r_{2}}\right) r^{2} d r=\int_{0}^{r_{2}} \frac{r_{2} \sin \left(\frac{r \alpha_{n}}{r_{2}}\right)}{r \alpha_{n}} r^{2} d r \\
&= \frac{r_{2}}{\alpha_{n}} \int_{0}^{r_{2}} r \sin \left(\frac{r \alpha_{n}}{r_{2}}\right) d r \\
&=\frac{r_{2}}{\alpha_{n}} \int_{0}^{\alpha_{n}} \frac{r_{2}}{\alpha_{n}} u \sin (u) \frac{r_{2}}{\alpha_{n}} d u \quad\left(u=\frac{r \alpha_{n}}{r_{2}}\right)
\end{aligned}
$$




$$
\begin{aligned}
& =\frac{r_{2}^{3}}{\alpha_{n}^{3}} \int_{0}^{\alpha_{n}} u \sin (u) d u \\
& =\left.\frac{r_{2}^{3}}{\alpha_{n}^{3}}(\sin (u)-u \cos (u))\right|_{0} ^{\alpha_{n}} \\
& =\frac{r_{2}^{3}}{\alpha_{n}^{3}}\left(\sin \left(\alpha_{n}\right)-\alpha_{n} \cos \left(\alpha_{n}\right)\right)=0 .
\end{aligned}
$$

Case III: $m=n, \alpha_{n}>0$

$$
\begin{array}{rl}
\int_{0}^{r_{2}} j_{0}^{2}\left(\frac{r \alpha_{n}}{r_{2}}\right) r^{2} & d r=\int_{0}^{r_{2}} \frac{r_{2}^{2} \sin ^{2}\left(\frac{r \alpha_{n}}{r_{2}}\right)}{r^{2} \alpha_{n}^{2}} r^{2} d r \\
& =\frac{r_{2}^{2}}{\alpha_{n}^{2}} \int_{0}^{r_{2}} \sin ^{2}\left(\frac{r \alpha_{n}}{r_{2}}\right) d r \\
& =\frac{r_{2}^{3}}{\alpha_{n}^{3}} \int_{0}^{\alpha_{n}} \sin ^{2}(u) d u \quad\left(u=\frac{r \alpha_{n}}{r_{2}}\right) \\
& =\left.\frac{r_{2}^{3}}{\alpha_{n}^{3}}\left(-\frac{\cos (u) \sin (u)}{2}+\frac{u}{2}\right)\right|_{0} ^{\alpha_{n}} \\
& =-\frac{r_{2}^{3}}{2 \alpha_{n}^{3}}\left(\cos ^{3}\left(\alpha_{n}\right) \sin \left(\alpha_{n}\right)+\alpha_{n}\right) \\
& =-\frac{r_{2}^{3}}{2 \alpha_{n}^{3}}\left(\left(\alpha_{n}-\alpha_{n} \cos ^{2}\left(\alpha_{n}\right)\right)+\alpha_{n}\right) \\
& =\frac{r_{2}^{3}}{2} \cos ^{2}\left(\alpha_{n}\right) . \\
& =\frac{r_{2}^{3}}{2 \alpha_{n}{ }^{2}}\left(1-\cos ^{2}\left(\alpha_{n}\right)\right) \\
& =\frac{r_{2}^{3}}{2 \alpha_{n}{ }^{2}} \sin ^{2}\left(\alpha_{n}\right) \\
& \cos ^{2}\left(\alpha_{n}\right) \\
&
\end{array}
$$

